\title{
Axial strain in GaAs/InAs core-shell nanowires
}

Andreas Biermanns, Torsten Rieger, Genziana Bussone, Ullrich Pietsch, Detlev Grützmacher, and Mihail Ion Lepsa

Citation: Appl. Phys. Lett. 102, 043109 (2013); doi: 10.1063/1.4790185

View online: https://doi.org/10.1063/1.4790185

View Table of Contents: http://aip.scitation.org/toc/apl/102/4

Published by the American Institute of Physics

\section{Articles you may be interested in}

Strain in semiconductor core-shell nanowires

Journal of Applied Physics 106, 053508 (2009); 10.1063/1.3207838

Band parameters for III-V compound semiconductors and their alloys

Journal of Applied Physics 89, 5815 (2001); 10.1063/1.1368156

Transport and strain relaxation in wurtzite InAs-GaAs core-shell heterowires

Applied Physics Letters 98, 152103 (2011); 10.1063/1.3579251

Residual strain and piezoelectric effects in passivated GaAs/AIGaAs core-shell nanowires

Applied Physics Letters 102, 191103 (2013); 10.1063/1.4803685

Faster radial strain relaxation in InAs-GaAs core-shell heterowires

Journal of Applied Physics 111, 044301 (2012); 10.1063/1.3684964

Strain influenced indium composition distribution in GaN/InGaN core-shell nanowires

Applied Physics Letters 97, 181107 (2010); 10.1063/1.3513345

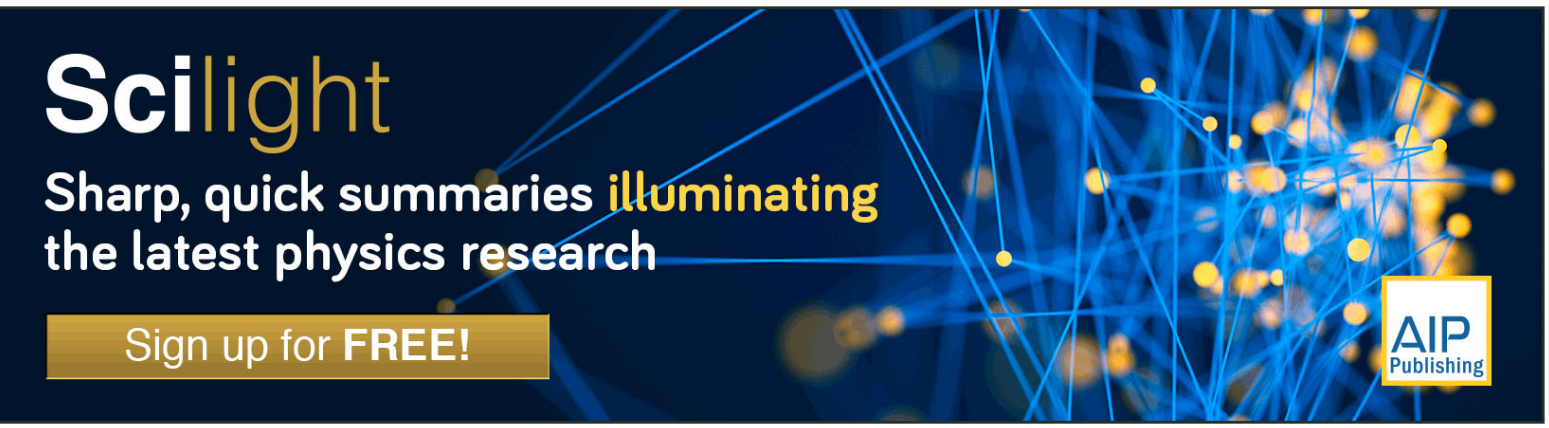




\title{
Axial strain in GaAs/lnAs core-shell nanowires
}

\author{
Andreas Biermanns, ${ }^{1, a)}$ Torsten Rieger, ${ }^{2,3}$ Genziana Bussone, ${ }^{1,4}$ Ullrich Pietsch, ${ }^{1}$ \\ Detlev Grützmacher, ${ }^{2,3}$ and Mihail Ion Lepsa ${ }^{2,3}$ \\ ${ }^{1}$ Universität Siegen, Festkörperphysik, 57068 Siegen, Germany \\ ${ }^{2}$ Peter Grünberg Institute (PGI-9), Forschungszentrum, 52425 Jülich, Germany \\ ${ }^{3}$ JARA - Fundamentals of Future Information Technology, 52425 Jülich, Germany \\ ${ }^{4}$ ESRF, 6 rue Jules Horowitz, BP220, F-38043 Grenoble Cedex, France
}

(Received 21 October 2012; accepted 18 January 2013; published online 30 January 2013)

\begin{abstract}
We study the axial strain relaxation in GaAs/InAs core-shell nanowire heterostructures grown by molecular beam epitaxy. Besides a gradual strain relaxation of the shell material, we find a significant strain in the GaAs core, increasing with shell thickness. This strain is explained by a saturation of the dislocation density at the core-shell interface. Independent measurements of core and shell lattice parameters by x-ray diffraction reveal a relaxation of $93 \%$ in a $35 \mathrm{~nm}$ thick InAs shell surrounding cores of $80 \mathrm{~nm}$ diameter. The compressive strain of $-0.5 \%$ compared to bulk InAs is accompanied by a tensile strain up to $0.9 \%$ in the GaAs core. (C) 2013 American Institute of Physics. [http://dx.doi.org/10.1063/1.4790185]
\end{abstract}

Semiconductor and in particular heterostructure nanowires (NWs) are promising building blocks for future electronic devices. For example, GaAs/AlGaAs core-shell NWs show enhanced photoluminescence intensity compared to pure GaAs NWs. ${ }^{1}$ Similarly, the electron mobility is increased by covering InAs NWs with InP shells. ${ }^{2}$ In both cases, the improved properties are attributed to the passivation of the NW surface. However, when an isolating large band gap semiconducting NW core (e.g., GaAs) is covered by a semiconducting low band gap shell (e.g., InAs), a tubelike conductive channel can be obtained. In this geometry, interesting electron interference phenomena are observed, ${ }^{3}$ which can be used in phase-based quantum devices for future fast and low-power consumption nanoelectronics. ${ }^{4}$

The interface quality between core and shell is important for device performance. However, mutual strain can arise at the heterointerface of lattice mismatched materials. The core-shell geometry allows only the coherent growth of small shell thicknesses, and for typical NW diameters, the critical thickness for dislocation free growth is slightly larger than for planar films. ${ }^{5}$ In the case of highly lattice mismatched III-V NW heterostructures, the strain relaxation of InAs/GaAs core-shell nanowires has been most extensively studied by transmission electron microscopy (TEM). The lattice mismatch between core and shell amounts in this case to $\delta a=\frac{a_{\mathrm{InAs}}-a_{\mathrm{GaAs}}}{a_{\mathrm{GaAs}}}=7.16 \% .{ }^{6}$ For this system, a fast strain relaxation of the shell along the radial direction has been reported, whereas a slower and gradual strain relaxation was observed in the axial direction, saturating at values of around $80 \% .^{7-9}$ In all the reports, the strain relaxation is commonly related only to the shell material, and a possible strain in the core region can hardly be accessed independently by electron microscopy. Complementary x-ray diffraction can be used to monitor the relaxation in core-shell nanowire heterostructures. ${ }^{10,11}$

In this paper, we study the axial strain relaxation in GaAs/InAs core-shell nanowire heterostructures grown by

\footnotetext{
a)Andreas.Biermanns@uni-siegen.de.
}

molecular beam epitaxy (MBE). Using x-ray diffraction, we are able to separate the strain within both core and shell regions. Besides a gradual relaxation of the shell, we find a significant strain in the GaAs core, increasing with shell thickness. The x-ray diffraction results are compared with TEM data obtained on the same NWs.

The GaAs NW cores were grown by MBE using the Gaassisted growth mode on a GaAs ( $\left.\begin{array}{lll}1 & 1 & 1\end{array}\right) \mathrm{B}$ surface covered by a thin $\mathrm{SiO}_{x}$ layer. ${ }^{12}$ The core growth was done for $45 \mathrm{~min}$ using a substrate temperature of $590^{\circ} \mathrm{C}$, a Ga rate of $0.075 \mu \mathrm{m} / \mathrm{h}$ and an As flux of $1 \times 10^{-6}$ torr. The resulting

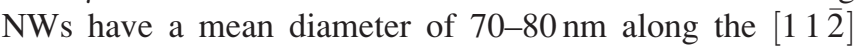
direction and a length of $1.2 \mu \mathrm{m}$. After the core growth, the samples were kept $10 \mathrm{~min}$ at the same constant temperature and As flux in order to consume the Ga droplet. The NW cores have mainly cubic zinc-blende (ZB) structure with few rotational twins except a short segment at the top, which is of the hexagonal wurtzite (WZ) phase and is created during the consumption of the liquid Ga droplet. ${ }^{12}$ Then, the substrate temperature was ramped down to $490^{\circ} \mathrm{C}$, and the growth of the InAs shell was initiated using the same As flux as for the core and an In rate of $0.1 \mu \mathrm{m} / \mathrm{h}$.

During the initial stage of InAs growth, InAs islands are formed on the core's [ $\left[\begin{array}{lll}1 & 1 & 0\end{array}\right]$ side facets, which coalesce and form a closed and smooth shell after reaching a thickness of $5 \mathrm{~nm} .^{13}$ Analysis of Moiré patterns obtained by TEM indicates a saturation of the axial relaxation at around $80 \%$ of the total mismatch, comparable to the value found in the InAs/GaAs core-shell case. ${ }^{7}$ A set of these NWs with closed InAs shell corresponding to growth times between 5 and 30 min has been selected for $\mathrm{x}$-ray diffraction experiments. Additionally, one sample was grown and investigated without the InAs shell.

Figure 1 shows scanning electron microscopy (SEM) pictures of the pure GaAs NWs (a) and the GaAs NWs covered by a $12 \mathrm{~nm}$ (b), $20 \mathrm{~nm}$ (c), and $35 \mathrm{~nm}$ (d) thick shell of InAs, respectively. For all samples, the shell thickness was determined by transmission electron microscopy after the transfer of NWs from the substrate to a TEM grid. Note that 

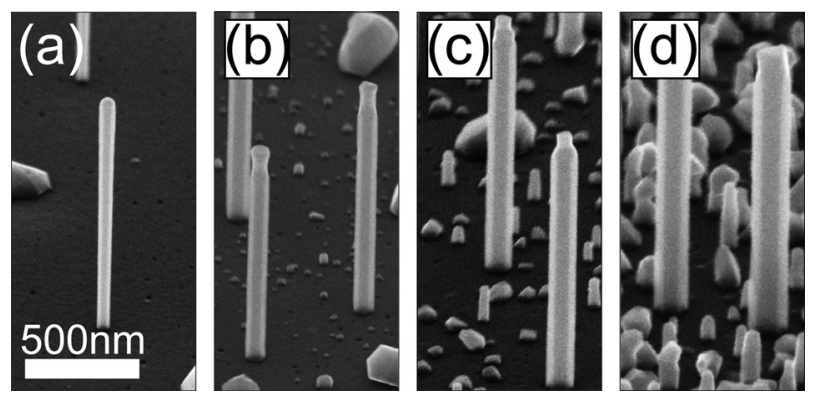

FIG. 1. SEM images of the inspected NWs. $1.2 \mu \mathrm{m}$ long GaAs NW cores (a) are covered by an InAs shell of $12 \mathrm{~nm}$ (b), $20 \mathrm{~nm}$ (c), and $35 \mathrm{~nm}$ (d), respectively. Together with the growth of the InAs shell, parasitic InAs growth occurs in between the initial NWs. (All images have the same scale.)

besides the increasing diameter of the NWs observed in SEM micrographs, parasitic growth of crystallites occurs in between the NWs.

High resolution $x$-ray diffraction experiments have been performed at the P08 beamline of the PETRA III synchrotron source. ${ }^{14}$ Here, the intensity distribution around the symmetric (111) reflections was measured in a coplanar diffraction geometry with the sample surface mounted horizontally. In addition, diffraction measurements using a nano-focussed $\mathrm{X}$-ray beam have been performed at the ID01 beamline of the ESRF synchrotron source in order to verify the attribution of the diffraction signals to nanowire core, shell, and crystallites, respectively. ${ }^{15}$

Figure 2(a) shows the intensity distribution around the GaAs (111) reflection (a reciprocal space map) of the bare GaAs NWs in terms of the momentum transfer of the diffracted $\mathrm{x}$-ray beam parallel to the surface $\left(\mathrm{q}_{x}\right)$ and perpendicular to it $\left(\mathrm{q}_{z}\right)$, respectively (cf. Fig. 2(e)). The GaAs substrate creates a sharp Bragg peak ("s") in reciprocal space, superimposed by a crystal truncation rod (CTR) originating from the surface of the substrate (vertical streak in Fig. 2(a)), as well as crossed streaks of intensity caused by the crystalmonochromator and the detector, respectively. ${ }^{16}$ Compared to that, the finite size and a possible random tilt of the GaAs nanowires lead to an elongated diffraction signal along the $\mathrm{q}_{x}$ direction in reciprocal space, allowing to separate the contributions from NW cores ("co") and substrate. For the pure GaAs NWs, the elongated signal coincides with the substrate signal, indicating that the NWs grow in the cubic ZB structure with bulk lattice parameter, in agreement with the TEM investigations. In addition, a weak signal is observed displaced by $\Delta q_{z} / q_{z} \approx-0.5 \%$ along the vertical direction. This signal can be attributed to the WZ segments at the top of the NWs, which are known to exhibit an increased lattice plane spacing along the growth direction compared to the $\mathrm{ZB}$ structure. $^{17,18}$

In reciprocal space, the length of the diffraction vector $q=\frac{2 \pi}{d}$ is inversely proportional to the spacing $d$ of the probed lattice planes. Hence, if a completely relaxed InAs shell is grown around the NW core, the larger (llll 111 ) lattice plane spacing of InAs leads to a Bragg peak located at smaller vertical momentum transfer compared to the GaAs core. However, if the InAs shell is subjected to compressive strain, the diffraction signal will be displaced along $\mathrm{q}_{z}$ towards the GaAs position. In Figs. 2(b)-2(d), the same region in reciprocal space around the GaAs ( $\left.\begin{array}{lll}1 & 1 & 1\end{array}\right)$ reflection is shown for the NWs covered with increasing InAs shell thickness. Figures 2(f)-2(h) show the corresponding region around the InAs $\left(\begin{array}{lll}1 & 1 & 1\end{array}\right)$ reflection, respectively. Most strikingly, with increasing shell thickness, the diffraction signal attributed to the GaAs core ("co" in Fig. 2(c)) shifts towards smaller momentum transfers, indicating an increase of the lattice plane spacing in the core material. This shift is indicated by arrows between Figs. 2(a)-2(c). Simultaneously, the peak broadens considerably along $\mathrm{q}_{z}$, until for a $35 \mathrm{~nm}$ thick shell, no distinct GaAs core reflection is observed anymore (see, Fig. 2(d)). In the latter case, the entire CTR of the substrate is surrounded by a strong diffuse scattering signal originating from the high surface coverage by parasitic crystallites, possibly shadowing the small core signal. For all shell thicknesses, the diffraction signal around the InAs (lll 111$)$ reflection (see, Figs. 2(f)-2(h)) consists of a superposition of two maxima, out of which one is located at the position of bulk ZB InAs ("cr") and the second one is displaced ("sh") towards larger $\mathrm{q}_{z}$, i.e., smaller lattice parameters. Spatially resolved nanodiffraction experiments using a focused $\mathrm{x}$-ray beam showed that the displaced GaAs and InAs diffraction signals originate from identical positions on the sample (the NWs with shell), whereas the relaxed InAs signal is caused by smaller crystallites in between. ${ }^{19}$ This compares well with the observation in Fig. 1, showing an increasing amount of parasitic InAs

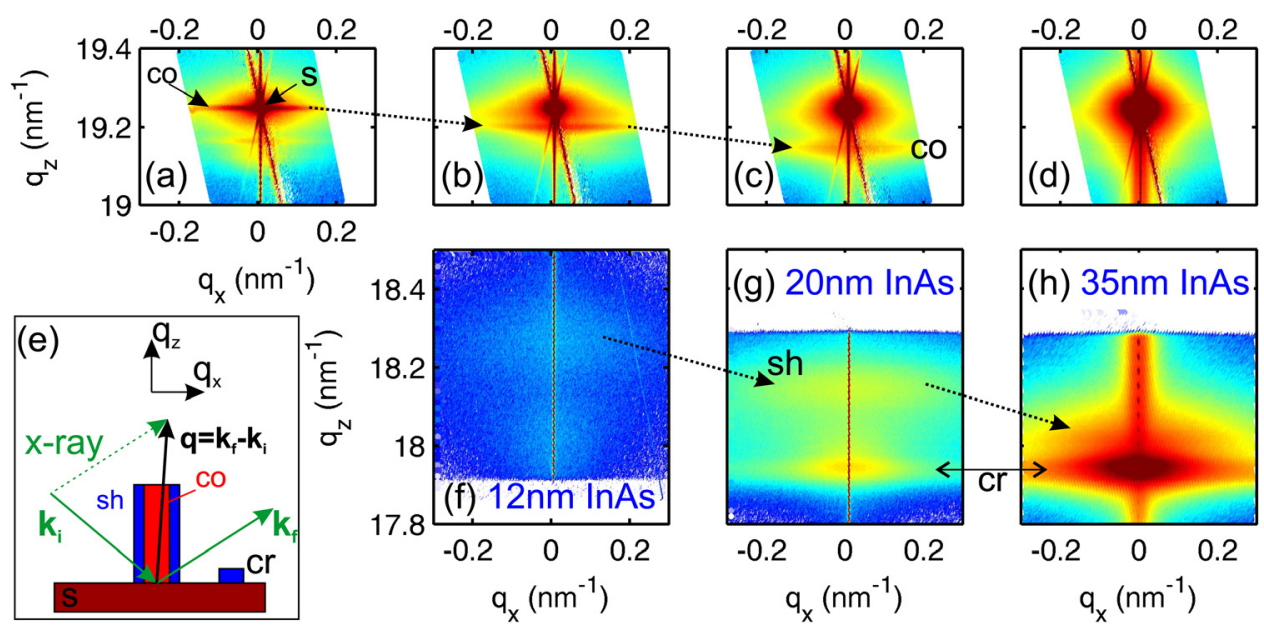

FIG. 2. X-ray reciprocal space maps around the GaAs ( $\left(\begin{array}{lll}1 & 1 & 1\end{array}\right)((\mathrm{a})-(\mathrm{d}))$ and InAs (1 11 1) ((f)-(h)) reflections of all investigated samples. With increasing shell thickness, the diffraction signal of the GaAs core (co) is displaced from the substrate (s), which is used as reference. Simultaneously, the shell (sh) shows a gradual relaxation and InAs crystallites (cr) grow. (e) shows a sketch of the diffraction geometry. (Logarithmic colorscale, from blue to red.) 
growth on the oxide covered surface during the shell deposition.

The mean strain values in GaAs core and InAs shell can be determined from their respective peak positions ("co" and "sh" in Fig. 2). Both are plotted in Fig. 3. The values are calculated with respect to the lattice plane spacing of unstrained material, taken from the position of the GaAs substrate reflection and the InAs crystallites measured simultaneously. The error bars are determined from the full width at half maximum of the diffraction signals along $\mathrm{q}_{z}$ and indicate the spread of the lattice parameter distribution rather than the experimental resolution $\left(\Delta a / a \approx 1 \cdot 10^{-4}\right)$. Whereas the InAs shell is under axial compressive strain, the GaAs core is tensile strained, increasing from $0.25 \%$ for a $12 \mathrm{~nm}$ thick InAs shell to $0.50 \%$ for a $20 \mathrm{~nm}$ thick InAs shell. Although the strain in the GaAs core could not directly be measured for the $35 \mathrm{~nm}$ shell, simulations of the elastic interaction between core and shell indicate an increase in the tensile strain in the core to around $0.9 \%$ in this case (see below).

The inset in Fig. 3 shows a TEM micrograph of a NW with $20 \mathrm{~nm}$ thick InAs shell. In the central region, a Moiré pattern is visible due to the overlapping lattices of core and shell. From the separation of the Moire fringes, the mismatch between both lattices can be obtained. For comparison, green triangles in Fig. 3 show the strain in the shell based on the TEM investigation, if only the deformation of the shell, but no change in the core lattice parameter is considered. The TEM data show a saturation of the strain in the shell around $(\Delta a / a)_{\text {shell }} \approx-1.5 \%$, corresponding to a relaxation of $80 \%$ relative to bulk values. TEM investigations have been performed for more combinations of shell thickness and core diameter, all showing a saturation with increasing shell thickness. ${ }^{13}$ Compared to this, the $\mathrm{x}$-ray data clearly show a smaller compressive axial strain in the shell, decreasing to $(\Delta a / a)_{\text {shell }} \approx-0.5 \%$ for $35 \mathrm{~nm}$ shell thickness. Considering the bulk lattice parameters of GaAs and InAs, this corresponds to a relaxation of $93 \%$, larger than observed by TEM. However, this discrepancy is resolved if the experimentally observed changes of both core and shell lattice parameters are taken into account, resulting in a relative difference of

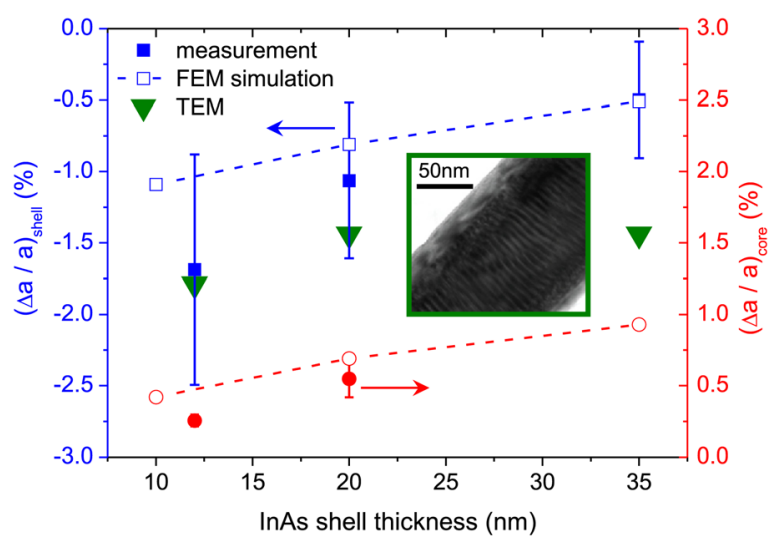

FIG. 3. Relative change of the core (red spheres) and shell (blue squares) lattice parameters along the growth direction as function of the shell thickness. The error bars measure the observed spread from the FWHM in Fig. 2. The green triangles represent the TEM results for the shell. The open symbols show results of the finite element simulation. the core and shell lattice parameters of $\approx 80 \%$ for all measured samples, in agreement with the TEM results.

The increasing strain in the core for larger shell thickness indicates that the plastic relaxation of the shell by inclusions of misfit dislocations is incomplete and does not reach $100 \%$. TEM investigations revealed the presence of both perfect dislocations and Frank partial dislocations at the interface. ${ }^{13}$ While the perfect dislocations with Burgers vector $\mathbf{b}=a / 2$ [110] can glide and, therefore, change their position during the shell growth, the Frank partial dislocations $(\mathbf{b}=a / 3$ [111]) cannot glide. Thus, the Frank partial dislocations will hinder the inclusion of additional misfit dislocations. Apart from that, the growth mechanism via islands and their coalescence can contribute also to the saturation of the dislocation density, as was observed in planar heteroepitaxy of InAs on GaAs $\left(\begin{array}{lll}1 & 1 & 1\end{array}\right) \mathrm{A}^{20}$ In the early stage of growth, the surface facets of the islands may introduce additional elastic stress relief accompanied by a reduced dislocation density compared to planar films. After coalescence, this situation will lead to a remaining effective lattice-mismatch and hence a stress at the heterointerface. If no additional defects are introduced at the interface, an increasing shell thickness will lead to a more efficient relaxation of the shell, accompanied by a higher strain in the core. Besides the increasing strain, the diffraction signal of the core indicates a rather homogeneous displacement throughout the entire core region.

In order to determine whether the observation of a homogeneous, increasing strain is consistent with the assumption of an incomplete plastic relaxation, the elastic interaction between core and shell has been simulated within a finite element method (FEM) approach in the COMSOL MULTIPHYSICS package. FEMs have been used before to study the in-plane strain distribution for the example of $\mathrm{GaAs} / \mathrm{GaP}$ core-shell NWs in Ref. 21 for a fixed combination of core and shell diameter. In our simulations, NW cores with $80 \mathrm{~nm}$ diameter and shell thicknesses of $10 \mathrm{~nm}, 20 \mathrm{~nm}$, and $35 \mathrm{~nm}$ have been modeled, taking into account the bulk elastic parameters of GaAs and InAs in the hexagonal NW geometry following the approach of Martin. ${ }^{22}$ For both core and shell,

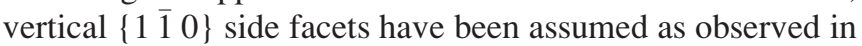
TEM investigations. Residual axial stress arising at the coreshell interface was simulated by applying a vertical expansion of the shell by means of a temperature increase, modeling the remaining effective mismatch at the heterointerface. In this approach, the microscopic structure of defects at the core-shell interface, which will vary among different NWs, is not explicitly taken into account and only the resulting average residual stress is considered. Here, only an initial axial mismatch (resulting in both axial and radial strains) has been considered, motivated by the fact that in InAs/GaAs coreshell NWs, the radial strain release occurs more rapidly than the axial one (see above). In the simulation, the residual mismatch was varied and the resulting average strain values in core and shell were compared with the experimental data. Considering all measured data points, a residual mismatch of $1.5 \%$ at the core-shell interface leads to the best agreement between simulation and experiment. These results are plotted as open symbols together with the experimental data in Fig. 3. For $35 \mathrm{~nm}$ shell thickness, we obtain a compressive axial strain of $(\Delta a / a)_{\text {shell }} \approx-0.5 \%$ 
in the shell, accompanied by a tensile axial strain of $(\Delta a / a)_{\text {core }} \approx 0.9 \%$ in the core. The general trend observed in the experiment can well be explained without the need of additional plastic relaxation for larger shell thickness and hence make clear the observed "saturation" of the axial relaxation. In addition, the simulation shows that the entire core region is considerably strained even for small shell thicknesses, in agreement with the displacement of the entire diffraction signal.

Comparing the absolute values and the general evolution of the strain in the core and shell with increasing shell thickness, small deviations are observed between simulation and measurement. Several factors may contribute to these deviations. First, the calculations for each sample have been performed for one selected shell and core diameter only, neglecting the random nature of NW growth, which gives small variations of the dimensions of both core and shell among different NWs on one sample. Second, only axial stress has been considered for all shell thicknesses (see above), neglecting possible tangential stresses around the NWs perimeter. Finally, bulk elastic constants have been considered for the simulation, although deviations of the elastic parameters are expected for small dimensions. ${ }^{23}$ This holds in particular for the thin InAs shell but also for the core diameter of $80 \mathrm{~nm}$. However, no conclusive picture about the changes in elastic properties and their dependence on geometric or size effects can be drawn to date. In the particular case of GaAs NWs, both an increase ${ }^{24}$ as well as a decrease $^{25}$ of Young's modulus compared to bulk GaAs has been reported.

To conclude, we have measured the absolute strains in GaAs/InAs core-shell NWs using x-ray diffraction, showing an increasing average relaxation of the shell together with an increasing strain in the core. This observation can be explained by a saturation of the dislocation density at the heterointerface, leading to an effective residual mismatch of $1.5 \%$ between core and shell. In addition, our measurements explain the observed "saturation" of axial relaxation in the shell by TEM methods. Both measurement and simulation show a relative difference in the lattice parameters of core and shell of $\approx 80 \%$ compared to the bulk lattice parameters. Due to the elastic interaction, this value does not change considerably even for large shell thickness, although the mean relaxation of the shell reaches a value of $93 \%$ (compared to bulk values) for $35 \mathrm{~nm}$ thickness. Here, only the axial relaxation has been discussed. A detailed study of the radial relaxation, which can be expected to occur more suddenly, will be the subject of future work.
We thank C. Deiter, O. Seeck, and F. Bertram from beamline P08, HASYLAB at DESY and G. Carbone and V. Jacques from beamline ID01, ESRF for support during the synchrotron experiments. We further thank R. Homs from ESRF for technical support.

${ }^{1}$ O. Demichel, M. Heiss, J. Bleuse, H. Mariette, and A. Fontcuberta i Morral, Appl. Phys. Lett. 97, 201907 (2010).

${ }^{2}$ X. Jiang, Q. Xiong, S. Nam, F. Qian, Y. Li, and C. M. Lieber, Nano Lett. 7, 3214 (2007).

${ }^{3}$ C. Blömers, T. Rieger, P. Zellekens, F. Haas, M. I. Lepsa, H. Hardtdegen, Ö. Gül, N. Demarina, D. Grützmacher, H. Lüth, and T. Schäpers, Nanotechnology 24, 035203 (2013).

${ }^{4}$ Nanotechnology: Information Technology I, edited by R. Waser (WileyVCH, Weinheim, 2008), Vol. 3.

${ }^{5}$ S. Raychaudhuri and E. T. Yu, J. Appl. Phys. 99, 114308 (2006).

${ }^{6}$ O. Madelung, Semiconductors: Data Handbook (Springer, Berlin, Heidelberg, 2004).

${ }^{7}$ K. L. Kavanagh, J. Salfi, I. Savelyev, M. Blumin, and H. E. Ruda, Appl. Phys. Lett. 98, 152103 (2011).

${ }^{8}$ R. Popovitz-Biro, A. Kretinin, P. Von Huth, and H. Shtrikman, Cryst. Growth Design 11, 3858 (2011).

${ }^{9}$ K. L. Kavanagh, I. Saveliev, M. Blumin, G. Swadener, and H. E. Ruda, J. Appl. Phys. 111, 044301 (2012).

${ }^{10}$ I. A. Goldthorpe, A. F. Marshall, and P. C. McIntyre, Nano Lett. 8, 4081 (2008).

${ }^{11}$ M. Keplinger, T. Mårtensson, J. Stangl, E. Wintersberger, B. Mandl, D. Kriegner, V. Holý, G. Bauer, K. Deppert, and L. Samuelson, Nano Lett. 9, 1877 (2009).

${ }^{12}$ T. Rieger, S. Heiderich, S. Lenk, M. I. Lepsa, and D. Grützmacher, J. Cryst. Growth 353, 39 (2012).

${ }^{13}$ T. Rieger, M. Luysberg, T. Schäpers, D. Grützmacher, and M. I. Lepsa, Nano Lett. 12, 5559 (2012).

${ }^{14}$ O. H. Seeck, C. Deiter, K. Pflaum, F. Bertam, A. Beerlink, H. Franz, J. Horbach, H. Schulte-Schrepping, B. M. Murphy, M. Greve et al., J. Synchrotron Radiat. 19, 30 (2012).

${ }^{15}$ A. Biermanns, S. Breuer, A. Davydok, L. Geelhaar, and U. Pietsch, J. Appl. Crystallogr. 45, 239 (2012).

${ }^{16}$ U. Pietsch, V. Holý, and T. Baumbach, High-Resolution X-Ray Scattering: From Thin Films to Lateral Nanostructures (Springer, New York, 2004).

${ }^{17}$ C. Panse, D. Kriegner, and F. Bechstedt, Phys. Rev. B 84, 075217 (2011).

${ }^{18}$ A. Biermanns, S. Breuer, A. Davydok, L. Geelhaar, and U. Pietsch, Phys. Status Solidi (RRL) 5, 156 (2011).

${ }^{19}$ See supplementary material at http://dx.doi.org/10.1063/1.4790185 for a description of the nanodiffraction experiment, showing the spatial distribution of the different diffraction signals.

${ }^{20}$ H. Yamaguchi, J. G. Belk, X. M. Zhang, J. L. Sudijono, M. R. Fahy, T. S. Jones, D. W. Pashley, and B. A. Joyce, Phys. Rev. B 55, 1337 (1997).

${ }^{21}$ J. Grönqvist, N. Sondergaard, F. Boxberg, T. Guhr, S. Åberg, and H. Q. Xu, J. Appl. Phys. 106, 053508 (2009).

${ }^{22}$ R. M. Martin, Phys. Rev. B 6, 4546 (1972).

${ }^{23}$ C. Q. Chen, Y. Shi, Y. S. Zhang, J. Zhu, and Y. J. Yan, Phys. Rev. Lett. 96, 075505 (2006).

${ }^{24}$ Y. B. Wang, L. F. Wang, H. J. Joyce, Q. Gao, X. Z. Liao, Y. W. Mai, H. H. Tan, J. Zou, S. P. Ringer, H. J. Gao et al., Adv. Mater. 23, 1356 (2011).

${ }^{25}$ P. Alekseev, M. Dunaevskii, A. Stovpyaga, M. Lepsa, and A. Titkov, Semiconductors 46, 641 (2012). 